\title{
Transmission Line Model for Patch Antenna on Metameterial Substrate
}

\author{
Rashmita Kumari Panigrahy \\ Electronic Science Department Berhampur University Bhanja Bihar,Berhampur, Odisha-760007,INDIA
}

Abstract: A simple transmission line model for a patch antenna on a metameterial substrate is proposed. The results are compared with those from FDTD model and found to have reasonable matching.

Keywords: FDTD; Metamaterial; Microstrip Antenna; Transmission Line Model

\section{INTRODUCTION}

Planar antennas based on Metamaterials are becoming popular in the antenna community. However, it is difficult to get a simple model for such antenna, since the metamaterial is dispersive in nature. Therefore, for analysis one has to depend on numerical models like the FDTD [1] or FEM [2]. It is almost impossible to start any elementary level project work for a student, unless there is a sophisticated CAD package, which are very costly. To avoid such problems, we propose a simple transmission line model for a metamaterial based patch antenna considering a sandwich of a Double Negative (DNG) material between two Double Positive (DPS) materials along the patch length. The DNG is assumed to obey the non-lossy Drude model [3] for its permeability and permittivity.

\section{PROPOSED MODEL}

Figure 1 shows the cross section of the planar antenna. In this antenna three substrates, two normal dielectrics

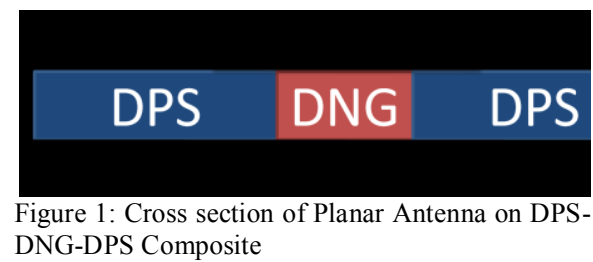

sandwiching a DNG are on a ground plane. The conducting patch sits on these substrates.

Using transmission line model, a rectangular patch antenna is modeled as two admittance slots at the two terminating ends of a transmission line. In such a model, the dielectric between the conducting patch and the ground plane is homogenous. Therefore, the transmission line connecting the two admittance slots is also considered to be a homogenous transmission line of characteristic admittance $\mathrm{Y}_{0}$. However, in the present case there is inhomogeneity in the dielectric material between the conducting patch and the ground plane. Therefore, it is natural to model each portion of dielectric as a separate transmission line and connect them in parallel. The structure under consideration (figure 1), suggests three sections of transmission lines (figure 2).

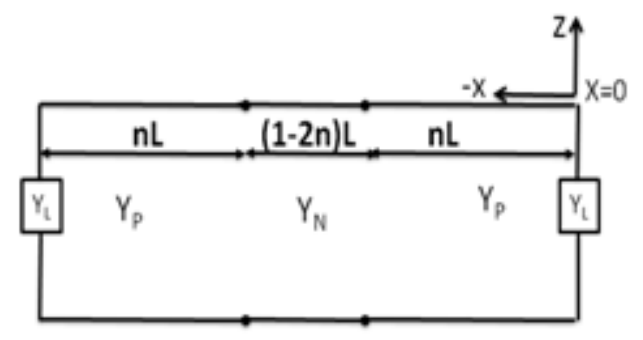

Figure 2: Proposed Transmission Line Model

The length of the conducting patch is ' $L$ '. The fraction of the patch length on the DNG material is $(1-2 n)$. The dielectric and magnetic constants of the substrate, under non-lossy Drude model consideration, are given as

$\varepsilon(x)=\left\{\begin{array}{cc}\varepsilon_{N}=1-\left(\frac{\omega_{e}}{\omega}\right)^{2} & (1-n) L \leq x \leq-n L \\ \varepsilon_{r} & \text { Otherwise }\end{array}\right.$ 
$\mu(x)=\left\{\begin{array}{cc}\mu_{N}=1-\left(\frac{\omega_{m}}{\omega}\right)^{2} & (1-n) L \leq x \leq-n L \\ 1 & \text { Otherwise }\end{array}\right.$

The loads at the two ends are expressed as [4]

$Y_{L}=G+j B$

$G=I / 120 \pi^{2}$

$I=-2+\cos X+X \operatorname{si}(\mathrm{X})+\frac{\sin \mathrm{X}}{\mathrm{X}}$

$X=k_{0} W$

$k_{0}=\omega / c$

$\operatorname{si}(X)=\int_{0}^{x} \frac{\sin y}{y} d y$

$B=\frac{W}{120 \lambda_{0}}\left[1-0.636 \ln \left(\frac{2 \pi h}{\lambda_{0}}\right)^{2}\right] \frac{h}{\lambda_{0}}<0.1$

To keep derivations simple we will consider feeding at the radiating edge. To find the input impedance at $x=-L$, first we transfer the load $Y_{L}$ at $x=0$ to admittance $Y_{I}$ at $x=-n L$. Then this impedance is transferred to admittance $Y_{2}$ at $x=-(1-n) L$ over a distance of $(1-2 n) L$. Finally this admitance is transferred to the input admittance $Y_{i n}$ at $x=L$ over a distance of $n L$. Therefore, the input admittance shall be given in terms of the admittances $Y_{L}, Y_{1}$ and $Y_{2}$.

$$
\begin{aligned}
& Y_{i n}=Y_{L}+Y_{d} \frac{Y_{2}+j Y_{d} \tan \left(\beta_{d} n L\right)}{Y_{X+j Y_{2} \tan \left(\beta_{d} n L\right)}} \\
& Y_{2}=Y_{N} \frac{Y_{1}+j Y_{N} \tan \left(\beta_{N}(1-2 n) L\right)}{Y_{N}+j Y_{1} \tan \left(\beta_{N}(1-2 n) L\right)} \\
& Y_{1}=Y_{d} \frac{Y_{L}+j Y_{d} \tan \left(\beta_{d} n L\right)}{Y_{d}+j Y_{L} \tan \left(\beta_{d} n L\right)} \\
& Z_{d}=\frac{1}{Y_{d}}=\frac{120 \pi}{\sqrt{\varepsilon_{e f f}}\left[\frac{W}{h}+1.393+0.667 \ln \left(\frac{W}{h}+1.444\right)\right]} \\
& Z_{N}=\frac{1}{Y_{N}}=\sqrt{\frac{\mu_{N}}{\varepsilon_{N}}}\left[\frac{\left.1 \frac{W}{h}+1.393+0.667 \ln \left(\frac{W}{h}+1.444\right)\right]}{2}\right. \\
& \varepsilon_{e f f}=\frac{\varepsilon_{r}+1}{2}+\frac{\varepsilon_{r}-1}{2} \frac{1}{\sqrt{1+12 h / W}} \\
& \beta_{d}=\frac{\omega \sqrt{\varepsilon_{e f f}}}{c} ; \quad \beta_{N}=\frac{\mp \omega \sqrt{\varepsilon_{N} \mu_{N}}}{c}
\end{aligned}
$$

$\mathrm{T}$ he $\frac{c}{\mp}$ sign in equation (16) is to indicate that the DNG can give $\bar{\mp}$ values for dielectric and magnetic constants at different frequencies. When both dielectric and magnetic constants are negative, the - sign is to be chosen. Otherwise the + sign is taken in the calculation.

\section{RESULTS AND DISCUSSIONS}

From the derivations, it is clear that the maximum value for $n$ shall be 0.5 while 0.0 shall be the minimum value. The former indicates absence of DNG material and the later indicates absence of DPS material. The developed model is applied with $f_{e}=1.6 \mathrm{GHz}$ and $f_{m}=0.8 f_{e}$. The substrate thickness is taken to be $2 \mathrm{~mm}$ and dielectric constant is 2.2 . The patch length is $36 \mathrm{~mm}$ and width is $40 \mathrm{~mm}$. The resonant frequency for this antenna shall be $2.8 \mathrm{GHz}$. Figure 3 shows the variation in impedance over a range of $2 \mathrm{GHz}$ to $10 \mathrm{GHz}$ for $n=0.1$.

Figure 4 shows the variations in impedance for $n=0.2,0.3$ and 0.4 .

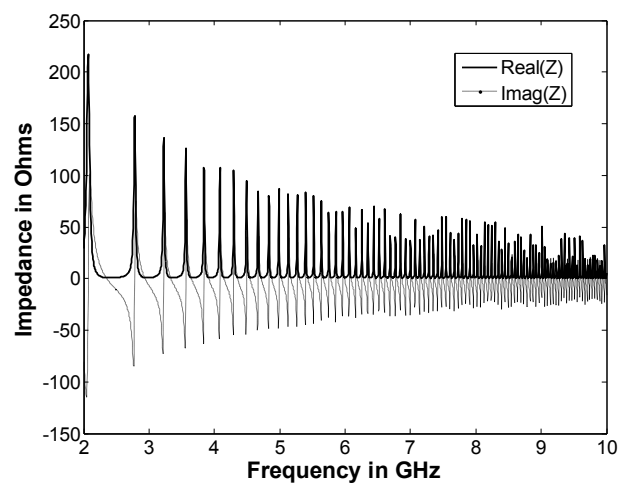

Figure 3: Variation of Impedance for $n=0.1$ 

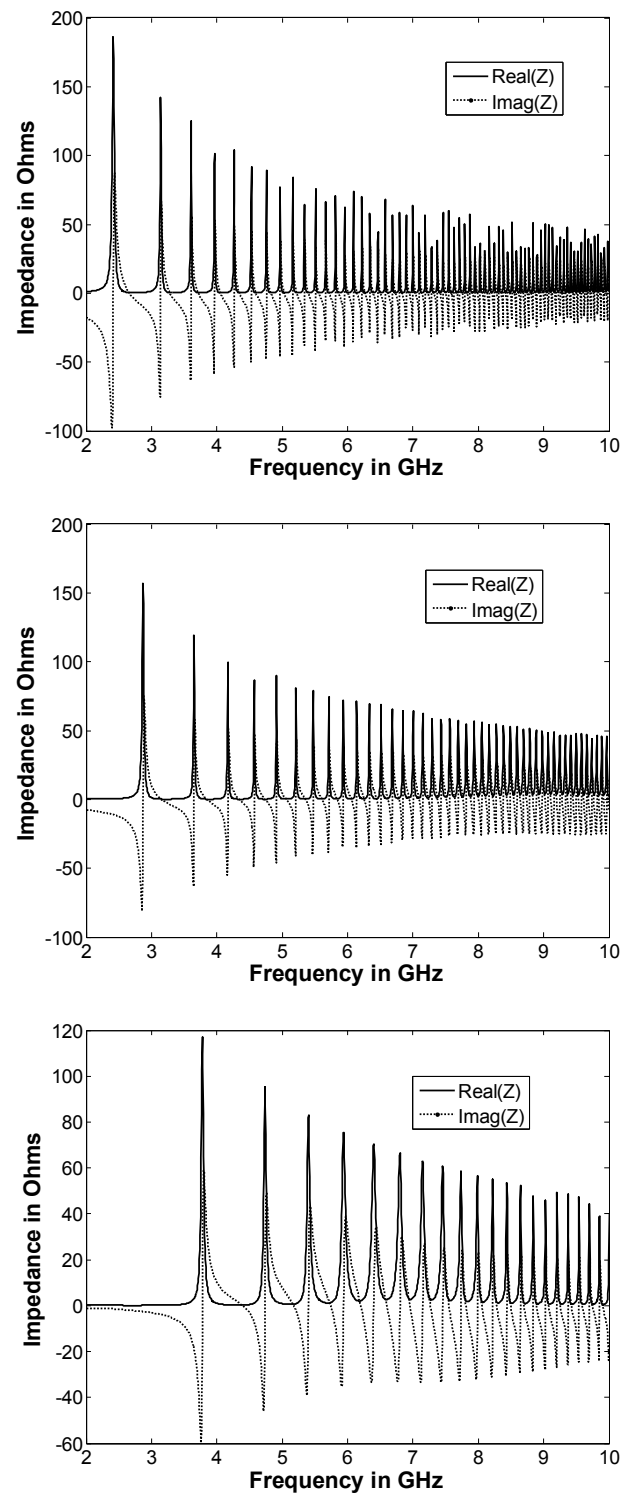

Figure 4: Impedance variations for $n=0.2$ (upper), 0.3 (middle) and 0.4 (lower)

From figures 2 and 3 it is observed that when $n$ is smaller, there are more number of resonances. The resonance peaks occur periodically but they are not exhibiting harmonic relation. A close observation also shows that the resonance peaks are closely spaced at higher frequencies than at lower frequencies. It suggests that the introduction of a DNG material into a DPS substrate results in multiple resonances spaced quasi-log periodically in frequency. The difference between the neighbouring resonance peaks is not very high. Therefore, this type of antenna is a good candidate for multi-frequency operation.

In the figure 5 , the resonance characteristic for $n=0.5$ is compared with the case when DNG is absent. 

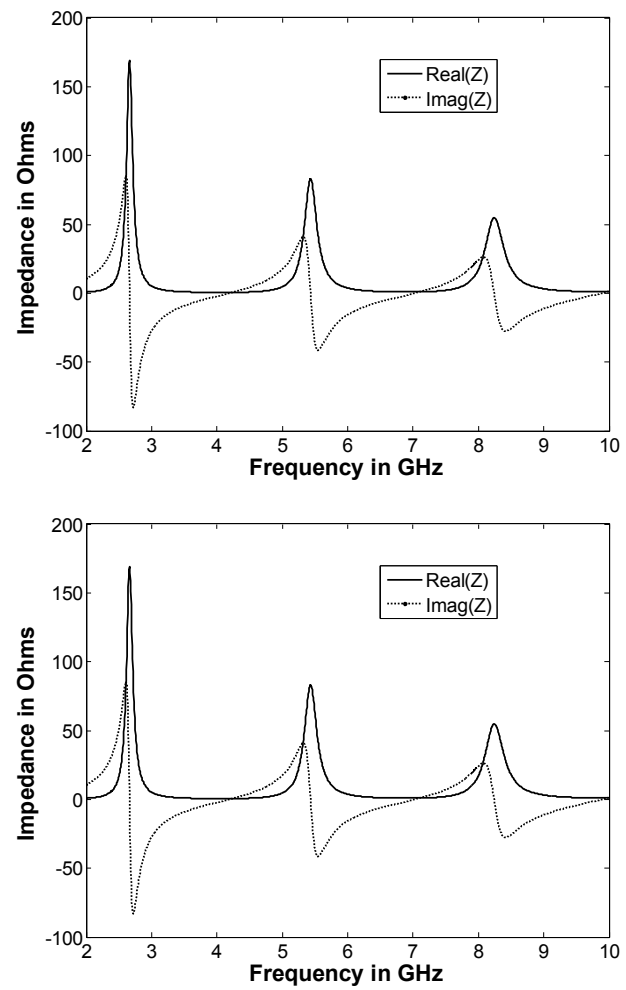

Figure 5: Impedance Characteristics for $n=0$ (upper) and in absence of DNG (lower)

It is observed that the plots in both the cases are completely identical. Also the harmonic relationship between the resonant frequencies is clearly evident.

\section{CONCLUSION}

A simple formula based on transmission line is proposed for calculating the input impedance of a rectangular patch antenna on a DPS-DNG-DPS longitudinal lattice. Using this formula, it is observed that a patch antenna shows multi-frequency characteristics for such an antenna. Also the resonant frequency distribution is harmonic. As the portion occupied by the DNG increases, the number of resonant peaks also increases and the first peak gets pushed downward in frequency. The validity of the model is also suggested from the comparison of the results for $n=0.5$ and pure DPS substrate.

\section{REFERENCES}

[1] K. P. Prokopidis, T. D. Tsiboukis, "The Effect of Substrate Dispersion on operation of square microstrip antennas," IEEE Trans. On Mag., Vol. 42, No. 4, pp. 603-606, 2006.

[2] S. N. Makarov, S. D. Kulkarni, A. G. Marut, L. C. Kempel, "Method of moments solution for a printed patch/slot antenna on a thin finite dielectric substrate using the volume Integral equation," IEEE Trans. On Ant. \& Prop., Vol. 54, No. 4, pp. 1174-1184, 2006.

[3] R. W. Ziolkowski, A. Erentok, "Metamaterial-based efficient electrically small antennas," IEEE Trans. On Ant. \& Prop., Vol. 54, No. 7, pp.2113-2130, 2006.

[4] C. A. Balanis, Antenna Theory: Analysis and Design, 2nd ed. New York: Wiley, 1997. 